\title{
Improving Learning Algorithm Performance for Spiking Neural Networks
}

\author{
Qiang Fu' ${ }^{1}$, Yuling Luo ${ }^{*}$, Junxiu $\mathrm{Liu}^{1}$, Jinjie Bi ${ }^{1}$, Senhui Qiu ${ }^{1,2}$, Yi Cao ${ }^{3}$, Xuemei Ding ${ }^{4,5}$ \\ ${ }^{1}$ Guangxi Key Lab of Multi-Source Information Mining \& Security, \\ Faculty of Electronic Engineering, Guangxi Normal University, Guilin, China, 541004 \\ ${ }^{2}$ Guangxi Experiment Centre of Information Science, Guilin, China \\ ${ }^{3}$ Department of Business Transformation and Sustainable Enterprise, Surrey Business School, \\ University of Surrey, Surrey, UK \\ ${ }^{4}$ Faculty of Software, Fujian Normal University, Fuzhou, China, 350108 \\ ${ }^{5}$ School of Computing and Intelligent Systems, Faculty of Computing and Engineering, Ulster University, UK, BT48 7JL \\ *Email: yuling0616@mailbox.gxnu.edu.cn
}

\begin{abstract}
This paper proposes three methods to improve the learning algorithm for spiking neural networks (SNNs). The aim is to improve learning performance in SNNs where neurons are allowed to fire multiple times. The performance is analyzed based on the convergence rate, the concussion condition in the training period and the error between actual output and desired output. The exclusive-or (XOR) and Wisconsin breast cancer (WBC) classification tasks are employed to validate the proposed optimized methods. Experimental results demonstrate that compared to original learning algorithm, all three methods have less iterations, higher accuracy, and more stable in the training period.
\end{abstract}

Keywords-spiking neural network; optimization method; learning performance

\section{INTRODUCTION}

Recently, spiking neural network (SNN) is generally regarded as the third generation of artificial neural networks [1], and consists of many neurons. In SNN, the neurons communicate with each other via firing spikes [2], [3] and fulfil tasks via appropriate learning algorithms [4]. The learning algorithms are usually divided into supervised leaning and unsupervised learning. In supervised leaning, the most commonly utilized algorithm is based on back propagation. A popular back propagation learning algorithm of SpikeProp was proposed in the approach of [5]. This algorithm allows multiple delayed synapses in SNN. However, the neurons only spike one time and the learning performance is not commendable. Therefore, some extended algorithms have been developed to improve the performance of SpikeProp. In the approaches of [6]-[9], back propagation with momentum, QuickProp and heuristic rules are used to improve the learning performance of SNNs respectively. Another back propagation algorithm allows neurons emitting multiple times [10], which is a modified version of SpikeProp. Even so, the learning performance can be still improved, e.g. it needs to iterate around 1,000 times (which is too long) to solve Exclusive-or (XOR) task when the learning rate is set to a small value (0.1). Therefore, the aim of this work is to improve the learning performance and three methods are proposed to improve the original algorithm [10].

The rest of this paper is organized as follows: Section 2 presents the spiking neuron model, SNN architecture and back propagation algorithm. Section 3 provides the improved methods for original algorithm. The experimental results are given in Section 4 and Section 5 concludes this paper.

\section{The NEURON MODEL AND LEARNING ALGORITHM}

In this section, the spiking neuron model and learning algorithm are introduced. A neuron is the basic unit of neural networks and the neuron model is an important component for SNN. The neurons connect with each other via synapses. The learning algorithm is used to adjust the synapse weights when neurons activate.

\section{A. Spiking Neuron Model}

The most commonly used model, namely Spike Response Model (SRM) [1], is used in this work. Each neuron is associated with a membrane potential $\left(u_{j}\right)$, and a spike of post synapse neuron $(j)$ is dynamically generated at time $\left(t_{j}\right)$ when the potential goes above a specified threshold $(\vartheta)$. When a neuron spikes, the membrane potentials of all its associated post neurons are incremented by the weights of the respective connections [11]. The membrane potential $u_{j}$ is described as:

$$
\begin{gathered}
u_{j}(t)=\sum_{t_{j}^{(f)} \in \Gamma_{j}} \eta\left(t-t_{j}^{(f)}\right) \\
\sum_{i \in \Gamma_{j}} \sum_{t_{i}^{(g)} \in F_{i}} \sum_{k=1}^{l} w_{j i}^{k} \varepsilon\left(t-t_{i}^{(g)}-d^{k}\right)
\end{gathered}
$$

where $w_{j i}^{k}$ is the synaptic weight from pre-synapse neuron $i$ to post-synapse neuron $j$ with a delay of $d^{k}, \varepsilon(s)$ is the spike response function, and $\eta(s)$ is the refractoriness function, $F_{j}$ indicates the spike train of post-synaptic neuron, and $\Gamma_{j}$ is the set of pre-synaptic neurons. They are described respectively as:

$$
\begin{gathered}
\varepsilon(s)=\frac{s}{\tau} \exp \left(1-\frac{s}{\tau}\right) H(s) \\
\eta(s)=-\vartheta \exp \left(-\frac{s}{\tau_{r}}\right) H(s) \\
F_{j}=\left\{t_{j}^{(f)} \mid 1 \leq f \leq n\right\}=\left\{t \mid u_{j}(t)=\vartheta\right\}
\end{gathered}
$$




$$
\Gamma_{j}=\{i \mid i \text { is presynaptic to } j\}
$$

where $\tau$ is a time constant, $\tau_{r}$ is the time delay constant, $H(s)$ is the Heavy-side step function, $n$ is the number of spikes and the spike train is ordered chronologically. If $1 \leq$ $f<g \leq n$, then $t_{j}^{(f)}<t_{i}^{(g)}$.

\section{B. Network Architecture and Learning Algorithm}

The learning algorithm introduced in this section belongs to back propagation algorithm. It is based on a fully connected feed forward neural network as shown in Fig. 1. This SNN is a three-layer feed forward network with multiple delays and multiple synapses per connection. The first layer, named input layer, acts as the input of the network. In input layer, neurons fire predefined spike trains, but are not involve in real processing. The second layer is hidden layer. And the last one is output layer.

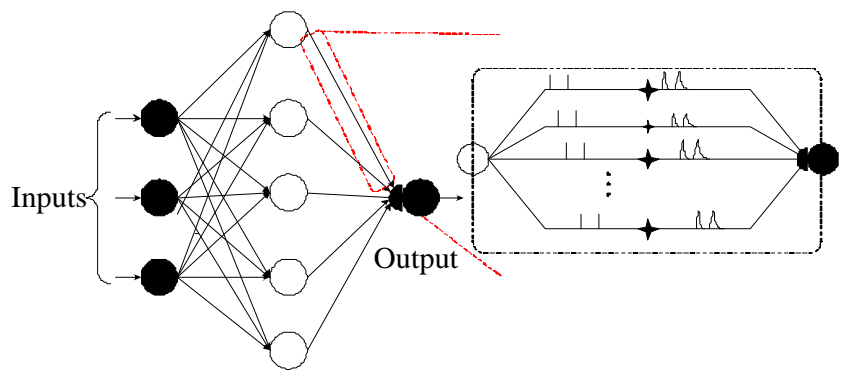

Figure 1. The feed forward architecture of SNN [5, 10, 12].

The detailed learning algorithm, which derived in $[5,10$, 13], uses the same rule as error back propagation. The measure function of this algorithm is determined by the difference between the desired output and actual output. It can be calculated by:

$$
E=\frac{1}{2} \sum_{j \in J}\left(t_{j}^{a}-t_{j}^{d}\right)^{2}
$$

where $t_{j}^{d}$ is desired firing time and $t_{j}^{a}$ is actual firing time at output neuron $j$.

To reduce the mean squared error, the weight $w_{i h}^{k}$ needs to be tuned. The rate of weight change can be calculated by:

$$
\Delta w_{i h}^{k}=-\eta \frac{\partial E}{\partial w_{i h}^{k}}
$$

where $\eta$ is the learning rate. The rule of renewing weight can be calculated by:

$$
w(n+1)=w(n)+\Delta w_{i h}^{k}
$$

More details of this learning algorithm can be in the approach of [13].

\section{IMPROVED METHODS}

As the learning performance is not commendable in original Booij's algorithm [10], three methods are employed to improve the original algorithm in this section. The improved methods include back propagation with inertia term, adaptive learning rate and the method of changing measure function.

\section{A. Inertia Term Adjustment}

As the original Booij's algorithm converges too slowly, an inertia term can be added to change the weight. This can be described as:

$$
\Delta w_{i h}^{k}=-\eta \frac{\partial E}{\partial w_{i h}^{k}}+\rho\left(w_{i h}^{k}-w_{i h}^{k-1}\right)
$$

where $\rho$ is the inertia factor, and its value is between 0 and 1 . The inertia term is not a fixed value. It can be adjusted as the synapse weight changes.

\section{B. Adaptive Learning Rate}

The learning rate can control the training time and accuracy. The convergence rate is also related to the learning rate. Although a large learning rate can speed up convergence, it usually leads to low accuracy. Therefore, a method of adaptive learning rate is can be used which is given by:

$$
\eta=a * e^{b * E}
$$

where $a$ is initial learning rate which is a small value. Once the mean squared error $(E)$ limits to $0, a$ will limit to $\eta$. The learning rate will be two times faster if multiply $a$ by a number that is greater than one. The parameter $b$ controls the value of learning rate. This method also can avoid the concussion condition in the training period because $\eta$ changes in the exponential manner.

\section{Changing Measure Function}

In original Booij's algorithm, the measure function is absolute error of desired firing time and actual firing time at output neuron. With the training time increases, it makes the difference of desired and actual firing time decrease. Then the convergence rate slows down. And the absolute error cannot represent the level of the relative error of the sample. To avoid these problems, the relative error can be employed. The relative error function can be calculated by:

$$
E=\frac{1}{2} \sum_{j \in J}\left(1-t_{j}^{d} / t_{j}^{a}\right)^{2}
$$

The above three methods are employed to improve original Booij's algorithm and enhance learning performance.

\section{EXPERIMENTAL RESULTS}

The proposed methods are analysed by applying them to the XOR task and Wisconsin breast cancer (WBC) classification task. The SNN architecture used in this work is shown by Fig. 1, where every connection consists of 16 synapses with a delay of 1 to $16 \mathrm{~ms}$. The parameters configuration of SNN are the same as [10]. The initial learning rate and time constant $(\tau)$ are set to 0.1 and 7, respectively. 


\section{A. XOR Task}

The XOR dataset is encoded by spike time in this work [10]. The binary 0's and binary 1's of inputs and outputs are encoded into firing time of input and desired output. For the inputs neurons, an input spike at $0 \mathrm{~ms}$ and $6 \mathrm{~ms}$ represent logic 0 and 1 , respectively. For outputs neuron, a spike at $16 \mathrm{~ms}$ and $10 \mathrm{~ms}$ represent logic 0 and 1 , respectively. For this task, the SNN architecture has 3 input neurons (including one bias neuron), 5 hidden neurons and 1 output neuron.

Fig. 2 shows the learning performances for the proposed three methods. Fig. 2(a) refers to original Booij's algorithm and it carries out 1016 epochs. Fig. 2(b) refers to the original algorithm with inertia term. It carries out 449 epochs. Fig. 2(c) refers to the algorithm with adaptive learning rate, which uses 81 epochs. Fig. 2(d) refers to the algorithm with relative error and it uses 62 epochs. It can be seen that concussion occurs in the Fig. 2(a) and Fig. 2(b) in the training period. However it does not occur in Fig. 2(c) and (d). Note that the convergence speeds of all three methods are faster than the original algorithm.
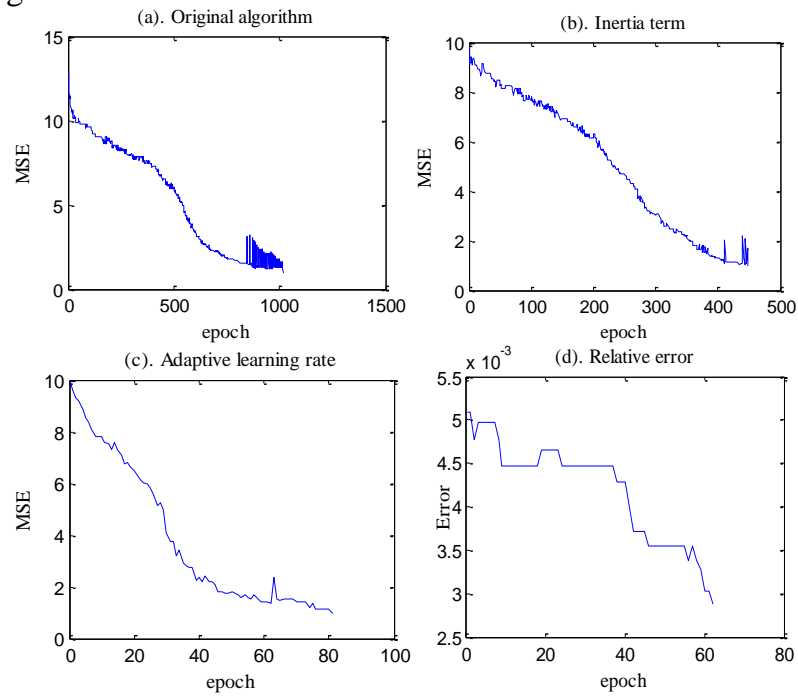

Figure 2. The relationship between epoch and error of XOR task with different learning methods.

\section{B. WBC Classification Task}

The WBC dataset uses breast cytology gained by fine needle aspirations in the University of Wisconsin Hospital and the results can be classified into benign or malignant cancer tumors [14]. This task is a binary classification problem. In the dataset, each sample consists of 9 attributes which measure different features of the cytology. Each feature is able to map to a linear spike train in the range of 1 to $10 \mathrm{~ms}$ directly. For the output neurons, the benign and malignant tumors can be coded as an output spike at time $16 \mathrm{~ms}$ and $17 \mathrm{~ms}$, respectively. The SNN for this task has 9 input neurons, 19 hidden neurons and 1 output neuron.

Fig. 3 shows the convergence curve for the proposed methods. Fig. 3(a) refers to original Booij's algorithm which carries out 230 epochs. Fig. 3(b) refers to the original algorithm with inertia term (109 epochs). Fig. 3(c) refers to the algorithm with adaptive learning rate ( 81 epochs). Fig. 3(d) refers to the algorithm with relative error (62 epochs). The detailed information is given by Table 1 .
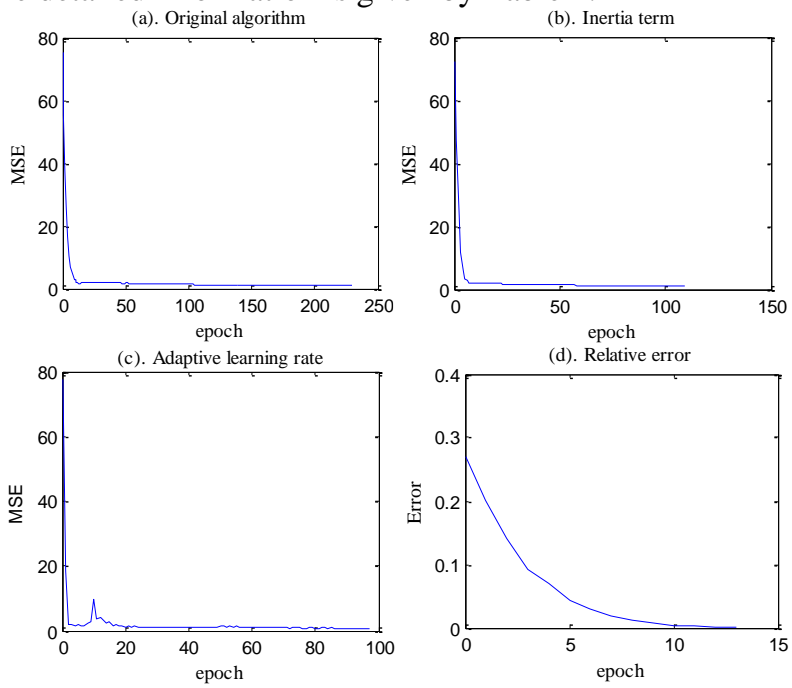

Figure 3. The relationship between epoch and error of WBC classification task with different methods.

TABLE I. THE DETAILED INFORMATION FOR WBC CLASSIFICATION TASK USING DIFFERENT ALGORITHMS.

\begin{tabular}{|c|c|c|}
\hline Algorithms & Epochs & Accuracy rate \\
\hline Original algorithm [10] & 230 & $79 \%$ \\
\hline Inertia term & 109 & $82 \%$ \\
\hline Adaptive learning rate & 98 & $90 \%$ \\
\hline Relative error & 13 & $85 \%$ \\
\hline
\end{tabular}

It can be seen that the convergence speed of all three methods, which proposed in this work, are faster than original algorithm. The highest classification accuracy is given by the method of adaptive learning rate, e.g. 90\%. For this method, the learning rate is related to the sum squared error $(E)$, which is a dynamic value and can guarantee the weight change function to get a suitable value.

\section{CONCLUSIONS}

In this paper, three methods are proposed to improve the learning performance of a gradient descent algorithm for the SNNs. The experiment of XOR task and WBC classification task are used for validation. The results showed that all three methods can speed up the convergence rate, and the methods of adaptive learning rate and relative error can avoid the concussion in the training period. Therefore, the methods proposed in this paper achieved a better performance than original algorithm [10].

\section{ACKNOWLEDGMENT}

This research was partially supported by the National Natural Science Foundation of China under Grants 61661008 and 61603104, the Guangxi Natural Science Foundation under Grants 2015GXNSFBA139256 and 2016GXNSFCA 380017, the funding of Overseas 100 Talents Program of Guangxi Higher Education, the Research Project of Guangxi 
University of China under Grant KY2016YB059, Guangxi Key Lab of Multi-source Information Mining \& Security under Grant MIMS15-07, the Doctoral Research Foundation of Guangxi Normal University, the grant from Guangxi Experiment Centre of Information Science, the Scientific Research Funds for the Returned Overseas Chinese Scholars from State Education Ministry, the Funds for Young Key Program of Education Department from Fujian Province, China (Grant No. JZ160425), Program of Education Department of Fujian Province, China (Grant No. I201501005).

\section{REFERENCES}

[1] W. Maass, "Networks of spiking neurons: The third generation of neural network models," Neural Networks, vol. 10, no. 9, pp. 1659$1671,1997$.

[2] J. Liu, J. Harkin, L. Mcdaid, D. M. Halliday, A. M. Tyrrell, and J. Timmis, "Self-repairing mobile robotic car using astrocyte-neuron networks," in International Joint Conference on Neural Networks, 2016, pp. 1-8.

[3] J. Liu, J. Harkin, L. P. Maguire, L. J. Mcdaid, and J. J. Wade, "SPANNER: A self-repairing spiking neural network hardware architecture," IEEE Trans. Neural Networks Learn. Syst., vol. PP, no. 99, pp. 1-14, 2017.

[4] X. Xie, H. Qu, G. Liu, and M. Zhang, "Efficient training of supervised spiking neural networks via the normalized perceptron based learning rule," Neurocomputing, vol. 241, pp. 152-163, 2017.
[5] S. M. Bohte, J. N. Kok, and H. La Poutré, "Error-backpropagation in temporally encoded networks of spiking neurons," Neurocomputing, vol. 48, no. 1-4, pp. 17-37, 2002.

[6] J. Xin and M. J. Embrechts, "Supervised learning with spiking neural networks," in International Joint Conference on Neural Networks, 2001, pp. $1772-1777$.

[7] S. McKennoch, D. L. D. Liu, and L. G. Bushnell, "Fast modifications of the SpikeProp algorithm," In: IEEE International Joint Conference on Neural Networks, pp. 3970-3977, 2006.

[8] R. A. Jacobs, "Increased rates of convergence through learning rate adaptation," Neural Networks, vol. 1, no. 4, pp. 295-307, 1988.

[9] B. Schrauwen, "Extending SpikeProp," in International Joint Conference on Neural Networks, 2004, pp. 471-476.

[10] O. Booij and H. Tat Nguyen, "A gradient descent rule for spiking neurons emitting multiple spikes," Inf. Process. Lett., vol. 95, no. 6, pp. 552-558, 2005.

[11] S. Sen, S. Venkataramani, and A. Raghunathan, "Approximate computing for spiking neural networks," in Design, Automation \& Test in Europe Conference \& Exhibition (DATE), 2017, pp. 193-198.

[12] X. Lin, X. Wang, and Z. Hao, "Supervised learning in multilayer spiking neural networks with inner products of spike trains," Neurocomputing, vol. 237, pp. 59-70, 2017.

[13] Y. Luo, Q. Fu, J. Liu, and J. Harkin, “An extended algorithm using adaptation of momentum and learning rate for spiking neurons emitting multiple spikes," in International Work-Conference on Artificial Neural Networks (IWANN), 2017, pp. 569-579.

[14] W. H. Wolberg and O. L. Mangasarian, "Multisurface method of pattern separation for medical diagnosis applied to breast cytology.," in Proceedings of the National Academy of Sciences, 1990, pp. 9193 9196. 